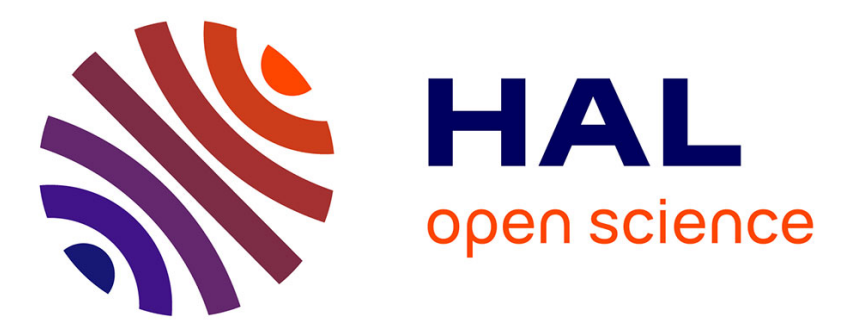

\title{
Designing Instructional Examples to Reduce Intrinsic Cognitive Load: Molar versus Modular Presentation of Solution Procedures
}

Peter Gerjets, Katharina Scheiter, Richard Catrambone

\section{To cite this version:}

Peter Gerjets, Katharina Scheiter, Richard Catrambone. Designing Instructional Examples to Reduce Intrinsic Cognitive Load: Molar versus Modular Presentation of Solution Procedures. Instructional Science, 2004, 32 (1-2), pp.33-58. 10.1023/B:TRUC.0000021809.10236.71 . hal-00197417

\section{HAL Id: hal-00197417 \\ https://telearn.archives-ouvertes.fr/hal-00197417}

Submitted on 14 Dec 2007

HAL is a multi-disciplinary open access archive for the deposit and dissemination of scientific research documents, whether they are published or not. The documents may come from teaching and research institutions in France or abroad, or from public or private research centers.
L'archive ouverte pluridisciplinaire HAL, est destinée au dépôt et à la diffusion de documents scientifiques de niveau recherche, publiés ou non, émanant des établissements d'enseignement et de recherche français ou étrangers, des laboratoires publics ou privés. 
Gerjets, P., Scheiter K. \& Catrambone, R. (2004). Designing Instructional Examples to Reduce Intrinsic Cognitive Load: Molar versus Modular Presentation of Solution Procedures. Instructional Science, 31(1-2), 33-58. This is a pre-print version of the article published in Instructional Science (C) Springer, 2004. The original publication is available at http://www.springerlink.com/content/jl14q60710wn5088/

Running head:

DESIGNING INSTRUCTIONAL EXAMPLES TO REDUCE INTRINSIC COGNITVE LOAD

Designing Instructional Examples to Reduce Intrinsic Cognitive Load:

Molar versus Modular Presentation of Solution Procedures

Peter Gerjets

Knowledge Media Research Center, Tuebingen, Germany

Katharina Scheiter

University of Tuebingen, Tuebingen, Germany

Richard Catrambone

Georgia Institute of Technology, Atlanta, USA

\begin{abstract}
Author Notes
The work reported in this paper was supported by the German Research Foundation DFG (Collaborative Research Center 378: Resource-adaptive Cognitive Processes) and by the Alexander von Humboldt-Foundation (TransCoop-Program). We thank Julia Schuh for helpful comments on an earlier version of this paper. Correspondence concerning this article should be addressed to Peter Gerjets, Knowledge Media Research Center, Konrad-Adenauer-Str. 40, 72072 Tuebingen, Germany. Electronic mail may be sent to p.gerjets@iwm-kmrc.de.
\end{abstract}




\begin{abstract}
It is usually assumed that successful problem solving in knowledge-rich domains depends on the availability of abstract problem-type schemas whose acquisition can be supported by presenting students with worked examples. Conventionally designed worked examples often focus on information that is related to the main components of problem-type schemas, namely on information related to problem-category membership, structural task features, and categoryspecific solution procedures. However, studying these examples might be cognitively demanding because they require learners to simultaneously hold active a substantial amount of information in working memory. In our research, we try to reduce intrinsic cognitive load in example-based learning by shifting the level of presenting and explaining solution procedures from a 'molar' view - that focuses on problem categories and their associated overall solution procedures - to a more 'modular' view where complex solutions are broken down into smaller meaningful solution elements that can be conveyed separately. We review findings from five of our own studies that yield evidence for the fact that processing modular examples is associated with a lower degree of intrinsic cognitive load and thus, improves learning.
\end{abstract}




\section{Designing Instructional Examples from a Cognitive Load Perspective}

It has often been argued that probably the most important prerequisite for successful problem solving consists in the availability of abstract problem-type schemas (Gick \& Holyoak, 1983; Reed, 1993), that is, representations of problem categories together with category-specific solution procedures. "Schemas are defined as mental constructs that allow patterns or configurations to be recognized as belonging to a previously learned category and which specify what moves are appropriate for that category” (Sweller \& Cooper, 1985, p. 60). Once a problem has been identified as belonging to a known problem category, the relevant schema is retrieved from memory, is instantiated with the information that is specific to the to-be-solved problem, and finally the category-specific solution procedure attached to the schema is executed in order to produce a solution to the problem (cf. Derry, 1989; VanLehn, 1989). Mayer (1981, p. 153) reports that "several groups of researchers have shown that students try to find out what 'type' of problem is presented and then to use a solution strategy appropriate for that type”.

Schema-based problem solving is considered to be very efficient and therefore often seen as a marking feature of experts’ problem solving (VanLehn, 1996). Accordingly, a substantial amount of research on skill acquisition has focused on the question of how such schemas can be acquired. An ubiquitous answer to this question is that studying concrete instances of problem categories (i.e., examples) is necessary for schema construction. In particular, worked examples (i.e., example problems together with step-by-step solutions) seem to play an important role in schema acquisition (cf. Atkinson, Derry, Renkl, \& Wortham, 2000; Sweller, van Merriënboer, \& Paas, 1998). Studying worked examples is superior to directly teaching abstract principles as well as to actively solving training problems - at least with regard to initial skill acquisition. This 'worked-example effect' is usually explained by pointing to the fact that studying worked 
examples imposes lower levels of cognitive load on the learner than solving training problems mainly because no extensive search processes with regard to the correct solution steps are involved. As a result, more cognitive resources might be left for the learner to engage in processes of schema construction. Moreover, it is assumed that studying worked examples (in contrast to attempting to solve training problems) focuses the learner's attention on information that is relevant to schema construction. For instance, studying worked examples might draw the learner's attention to structural task features that define to which problem category a particular problem belongs or it might draw the learner's attention to the solution rationale behind a category-specific solution procedure.

Conventional Example Design: Molar Presentation of Solution Procedures

Analyzing cognitive skill acquisition based on the notion of problem-type schemas quite naturally has specific implications for the design of instructional examples. Sweller et al. (1998) propose that “learners' attention must be withdrawn from processes not relevant to learning and directed toward processes that are relevant to learning and, in particular, toward the construction and mindful abstraction of schemas” (p. 264). For instance, if worked examples are intended to foster schema construction their design should focus on the information that is related to the main components of problem-type schemas, namely on information related to problem-category membership, structural task features, and category-specific solution procedures. These considerations fit rather well to the way instructional examples are conventionally designed - at least in textbooks on knowledge-rich and well-structured domains like physics, mathematics, or programming. Examples that can be found in these textbooks often tend to present solution procedures in a molar way as 'recipes' that are appropriate for particular problem categories. In order to enable learners to apply these 'recipes' the examples demonstrate how to categorize 
problems by considering multiple (and often abstract) structural task features. For instance, with regard to algebra word problems Reed (1999, p. 92) notes that "the solution of algebra word problems typically begins with the categorization of the problem based on the situation described in the problems.” (p. 92). Mayer (1981) extensively elaborated the categorical structure of word problems by collecting 1100 story problems from 10 major algebra textbooks used in California secondary schools and developing a taxonomy consisting of families, categories, and templates that describe the category membership of problems at different levels of abstraction. Atkinson and Catrambone (in press) note that mathematical problem solving is often characterized by 'computationally-friendly' molar solution approaches in which multiple solution steps are collapsed into a single formula that represents the solution procedure. These 'recipe-like' formulas allow one to easily calculate solutions by simply inserting the correct variable values.

To sum up, math textbooks often present mathematical problem solutions in a molar way and use 'category-focusing' instructional examples that are designed to illustrate how these 'recipes' are applied. However, formulas are usually restricted to solving a narrow range of problems that fall into predefined problem categories corresponding to the solution formula. Additionally, the strong focus on problem categories might cause learners to "memorize stereotypic solutions to problems based on their categorization” (Reed, 1999, p. 95). Thus, although schemas and problem categories are obviously very useful as a means for organizing knowledge representations and for guiding problem solving, they are not unequivocally advocated by math educators. For instance, Sowder (1985) argues that students should also be enabled to solve problems without relying on problem categories.

To illustrate the notion of problem-type schemas in greater detail and to elaborate on how conventional molar (or category-focusing) examples are usually designed, we will refer to the 
domain of probability word problems that is illustrated in Table 1 . The left column of Table 1 contains a conventionally designed molar solution for an example problem related to calculating complex event probabilities. The right column of Table 1 displays an alternative modular solution approach that we developed to improve example-based learning and that will be explained in greater detail below. Because we used problems like the one in Table 1 for experimentation in all studies reviewed in the second part of this paper, we will introduce the domain of calculating complex event probabilities in this section in some detail. Problems of calculating complex event probabilities are related to situations where the probability of selecting a particular configuration of elements randomly out of a set of elements has to be determined. A typical example includes calculating the probability of winning a lottery, for instance, calculating the probability of correctly guessing the six winning numbers out of a set of 49 numbers.

Four different problem categories are commonly distinguished in this area of probability theory (permutations and combinations, each with and without replacement) that differ with regard to two structural features. The first is whether the order in which elements are selected is important; the second is whether selected elements are replaced after being chosen. Depending on these two structural features different problems will require different formulas for their solution. Accordingly - like in many other mathematical areas - the calculation of complex event probabilities can be taught by means of category-specific solution formulas. The rationale of this approach is to divide the number of acceptable complex events by the number of possible complex events; category-specific solution formulas are used to calculate the number of possible complex events. The solution procedure based on this approach comprises four steps that are illustrated in the conventional molar example format in the left column of Table 1, namely, (1) identify task features, (2) apply formula, (3) insert values, and (4) calculate probability. 
---- Insert Table 1 here ---

This solution approach is a convenient and fast way of calculating complex event probabilities. The conventional molar example format illustrated in Table 1 might be well suited for conveying this approach by explaining how to categorize problems and how to apply category-specific solution formulas.

\section{Profitable Example Processing and Patterns of Cognitive Load}

When providing learners with conventional worked examples like the one presented in the left column of Table 1, instructors usually intend to trigger the construction of an appropriate problem-type schema that will allow learners to solve all problems that belong to the same problem category as the example problem. Research in the domain of learning from worked examples over the last 15 years has however demonstrated that the mere availability of conventional instructional examples does not seem to be sufficient to promote an adequate representation of problem categories, an understanding of category-specific solution procedures, and problem-solving transfer.

Rather, learners are often described as having difficulties identifying relevant information in worked examples (i.e., their structural task features) and as being distracted by the surface features of the examples (Ross, 1989). Furthermore, Renkl (1999) suggests that students often suffer from illusions of understanding when learning from worked examples. That is, they might have the false impression of having grasped the solution rationale of an example problem. Similarly, Catrambone (1998, p. 355) notes that learners "tend to form solution procedures that consist of a long series of steps - which are frequently tied to incidental features of the problems rather than more meaningful representations that would enable them to successfully tackle new problems”. That is, learners have difficulties generalizing solutions from examples to novel 
problems (Catrambone \& Holyoak, 1989; Reed, Dempster, \& Ettinger, 1985).

To sum up, numerous findings indicate that students experience serious difficulties in example-based learning resulting in the acquisition of rather shallow representations of problem categories and solution procedures.

Research on the role of example elaborations in learning has demonstrated that learners need to draw inferences concerning the structure of example solutions, the rationale behind solution procedures, and the goals that are accomplished by individual solution steps (e.g. by relating example-specific information to more abstract information; Chi, Bassok, Lewis, Reimann, \& Glaser, 1989; Pirolli \& Recker, 1994; Renkl, 1997) in order to overcome these difficulties. Example elaborations may in particular be helpful to foster learners' skills in solving novel problems that do not fall into known problem categories and that require an adaptation of procedures illustrated by worked examples.

Beyond example elaborations, learners have to engage in profitable processes of example comparison in order to notice structural features that differ among problem categories and that are shared by all problems within a category. If learners compare examples within and among problem categories with regard to their differences and similarities they might be more likely to identify the relevant features of worked examples and to avoid confusion due to the surface features of the examples (Cummins, 1992; Quilici \& Mayer, 1996).

Unfortunately, it has often been observed that learners do not spontaneously engage in these profitable processes of example elaboration and example comparison when studying worked examples (e.g., Chi et al., 1989; Gerjets \& Scheiter, 2003; Gerjets, Scheiter, \& Tack, 2000; Schuh, Gerjets, \& Scheiter, 2003). Rather, learners seem to need additional instructional support and carefully designed learning materials in order to make the most of instructional worked examples. 
To address the issue of developing improved instructional settings that ensure profitable processing of worked examples, we refer to the instructional-design framework provided by cognitive load theory (Sweller et al., 1998). According to this theory, constructing a problem-type schema might impose cognitive load on learners, that is, it demands working-memory resources because it requires learners to simultaneously process all information units that are to be integrated into that schema. These working-memory demands are particularly high for learners with low domain-specific prior knowledge. These learners lack complex knowledge structures that would otherwise help to increase the amount of information that can be held in working memory simultaneously by chunking individual knowledge elements into a single element. Within cognitive load theory, three types of cognitive load are distinguished:

- Intrinsic cognitive load: The number of elements that are to be integrated into a to-belearned schema and therefore have to be processed in working memory simultaneously is referred to as intrinsic cognitive load. Intrinsic cognitive load depends on the relational complexity of the to-be-learned content (so-called element interactivity) and the learner's degree of prior knowledge (i.e., schema availability). It is usually assumed in cognitive load theory that intrinsic cognitive load cannot be altered by instructional design.

Beyond intrinsic cognitive load there might be additional cognitive load due to the instructional presentation of the material and the activities learners are engaged in. This load can be influenced by instructional design and can be categorized according to whether it is beneficial for schema construction (i.e., germane cognitive load) or not (i.e., extraneous cognitive load).

- Germane cognitive load: When intrinsic task demands (resulting in intrinsic cognitive load) leave sufficient cognitive resources available, learners might "invest extra effort in processes that are directly relevant to learning, such as schema construction. These 
processes also increase cognitive load, but it is germane cognitive load that contributes to, rather than interferes with, learning” (Sweller et al., 1998, p. 264). Germane cognitive load is imposed by adding higher-level cognitive processes to the mere simultaneous activation of elements in working memory; these processes integrate the elements into a schema. In the case of learning from worked examples, germane cognitive load might be imposed onto the learner by cognitively demanding activities like example comparisons and example elaborations.

- Extraneous cognitive load: Extraneous cognitive load is the result of implementing "instructional techniques that require students to engage in activities that are not directed at schema acquisition” (Sweller, 1994, p. 299). For instance, these activities might comprise processes of finding, relating, or integrating particular pieces of information within instructional materials (whereas with a redesign such processing might not be required to the same degree). Extraneous cognitive load might impede learning, as it requires cognitive resources that can no longer be devoted to mindful cognitive processes that are associated with germane cognitive load. Furthermore, cognitive resources required by extraneous cognitive load might result in an overall cognitive load that exceeds the limits of working-memory capacity.

From a cognitive load perspective, an important objective of instructional design in example-based learning is to reduce extraneous cognitive load and to encourage learners to invest unused resources in higher-level cognitive processes such as example comparisons and example elaborations that are associated with germane cognitive load. Accordingly, several suggestions have been made on how to design instructional materials in order to foster a profitable utilization of worked examples and thus to improve the resulting pattern of cognitive load during learning. 
With regard to example comparisons it could be shown that the provision of multiple examples can support schema induction (Cummins, 1992). Additionally, providing multiple examples with different surface features might further improve example comparisons (Quilici \& Mayer, 1996). With regard to example elaborations it has been shown that training can be used to encourage learners to engage in self-explanations (Chi, de Leeuw, Chiu, \& LaVancher, 1994). Additionally, it has been shown that grouping solution steps according to their subgoals (Catrambone, 1998) is effective because it provides affordances for learners to self-explain the meaning of individual solution steps (Chi et al., 1989). Finally, completion problems (van Merriënboer, 1990) and fading procedures (Renkl \& Atkinson, 2003) have been introduced to prevent learners from being overly passive in studying instructional examples.

Nearly all instructional interventions conceived to improve learning from (conventionally designed) worked examples are intended to either increase germane cognitive load (i.e., profitable example processing) and/or to decrease extraneous cognitive load (i.e., activities not directed at schema acquisition). In our own research we try to go beyond this general rationale and to find a way to reduce intrinsic cognitive load in example-based learning by abandoning the molar structure of conventionally designed worked examples.

Reducing Intrinsic Cognitive Load in Example-based Learning

As mentioned before, cognitive load theory usually assumes that intrinsic cognitive load cannot be manipulated by instructional design because it depends directly on the number of elements that are to be integrated into a to-be-learned schema and therefore have to be processed in working memory simultaneously. According to the theory, this element interactivity depends only on the relational complexity of the to-be-learned content and on the learner's degree of prior knowledge (i.e., on schemas already available). However, the assumption that intrinsic cognitive 
load cannot be manipulated is not uncontroversial (cf. van Merriënboer, Kirschner, \& Kester, 2003). Different instructional approaches have been proposed that aim at reducing intrinsic cognitive load associated with learning materials.

- Part-whole sequencing: When the content of learning pertains to solving complex tasks, it is a well-known instructional approach to break down the complex task into simpler subtasks that can be conveyed separately. When learners have mastered the subtasks they may be instructed on how to solve the total complex task (cf. Gagné, 1962). This partwhole sequencing strategy is suitable for reducing intrinsic cognitive load. First the load associated with acquiring the component tasks is lower than the one that would be imposed by starting with acquiring the total complex task right from the beginning. Second, when learners are finally instructed on how to solve the total complex task later in the instructional sequence they will already possess a certain amount of domain-specific prior knowledge (in terms of schema availability) from their exposure to the component tasks. This prior knowledge will reduce the intrinsic cognitive load imposed by the need to finally acquire the skill of accomplishing the total complex task. However, it has to be noted that the part-whole sequencing strategy reduces the amount of intrinsic cognitive load during learning by changing the learning task in the early phases of the instructional sequence. So it might on the one hand be argued that this strategy does not predominantly change the cognitive load associated with a task but that it simply changes the task itself. On the other hand, the total complex task that is acquired later in the instructional sequence is the same task that would have been used without the part-whole sequencing strategy. From the latter perspective this instructional strategy does not change the learning task but instead improves learners' knowledge and skills before being confronted 
with the learning task and thereby reduces intrinsic cognitive load during learning due to an increased availability of related schemas.

- Simplified whole tasks: Van Merriënboer et al. (2003) argue that the fragmented approach of part-whole sequencing might make it difficult to integrate and coordinate subtasks into total complex tasks. Therefore, they advocate a different approach to lowering intrinsic cognitive load during learning, namely, to start learning with a simplified whole task with lower element interactivity. That is, the number of subtasks out of which the to-be-taught complex task consists is kept constant during training. However, each of these subtasks is first taught in a simplified version and then the difficulty of the subtasks and thereby of the whole task is gradually increased. As with part-whole sequencing, this approach might be considered as changing the learning task at the beginning of the instructional sequence by confronting the learners with simpler problems initially. It is only at the end of the instructional sequence - when learners already posses sufficient prior knowledge - that they are presented with the task in its full complexity. Again, the increased prior knowledge at that point in time results in a lower level of intrinsic cognitive load when learning how to accomplish the complex task.

- Modular presentation of solution procedures: Inspired by the aforementioned approaches to reduce the intrinsic cognitive load associated with learning from worked examples, we tried to design instructional examples that allow learners to start with a total complex task right from the beginning but nevertheless reduce intrinsic cognitive load during learning. Our approach was to develop a more modular solution procedure in examples that required a learner to keep only a limited number of elements active simultaneously in working memory. The basic idea behind this modular example format is to present solution 
procedures in a way that completely avoids references to conventional molar concepts like problem categories, clusters of structural task features, and category-specific solution procedures. It can be argued that these molar concepts - that refer to complex entities usually impose high levels of cognitive load onto the learner (for details see the next section). In contrast, modular examples focus on smaller meaningful solution elements and their relation to individual structural task features. This approach substantially decreases the number of elements that have to be considered at the same time. The modular examples that we constructed differ very much from a part-whole sequencing strategy because we convey right from the beginning how to solve total complex tasks rather than teaching how to solve smaller subtasks in isolation first. Furthermore, our approach is also different from using simplified whole tasks, because we do not alter the difficulty of learning tasks in the course of learning in order to reduce intrinsic cognitive load. Instead, we use exactly the same example problems for designing modular examples as we used for designing molar examples. The next section illustrates in greater detail how examples with a modular presentation of solution procedures might differ from examples with a conventional molar presentation of solutions.

\section{Designing Modular Worked Examples}

In the construction of modular worked examples we started with a careful analysis of the pattern of cognitive load imposed by conventional molar examples. A main feature of conventional examples is that they are designed to explain how to categorize problems according to multiple structural task features and how to apply category-specific solution formulas. Thus, molar examples usually demonstrate a convenient and fast way of solving problems that is quite similar to the approach a domain expert would choose for problem solving. However, a strong 
focus on problem categories might be rather overwhelming for novice learners because they might not possess sufficient prior knowledge (in terms of schema availability) to cope with the cognitive load imposed by this approach. A category-based approach requires learners to keep in mind all category-defining structural features of a problem before they are able to accurately decide on its problem category and the appropriate formula needed for its solution. Accordingly, studying molar examples requires that learners consider multiple structural task features at the same time in order to understand the problem's category membership.

As problems are grouped together in a problem category because they share a common solution procedure at some level of abstraction, the solution procedure associated with a problem category (i.e., applying an appropriate solution formula) can usually be characterized as a molar entity. For instance, in mathematical problem categories, multiple solution steps are frequently collapsed into a single complex formula that represents the solution and can be used as a recipe that allows one to calculate solutions in a fast and computationally convenient way. However, one has to consider that during schema acquisition all information units that are to be integrated into that schema have to be simultaneously activated in working memory (Sweller et al., 1998). Therefore, molar examples will result in a high level of intrinsic cognitive load - in particular for novice learners - depending on the number of structural task features that have to be kept in mind concurrently and depending on the complexity of the solution formula needed. As already mentioned before, a substantial amount of intrinsic cognitive load might prevent learners from engaging in profitable processes of elaborating or comparing examples that are necessary to overcome shallow representations of problems and their solution.

The modular example format that we developed in response to this analysis was intended to impose less intrinsic cognitive load on learners by avoiding the need for learners to consider 
multiple structural task features or multiple solution steps simultaneously. The rationale in the construction of this example format was to isolate task features and meaningful solution elements that can be conveyed and understood separately, thereby reducing intrinsic cognitive load. Besides reducing cognitive load, a second advantage of this approach is that it might allow learners to understand relations below the category level, that is, relations holding irrespective of category membership such as relations between individual structural task features and individual solution steps. As a result, learners might acquire meaningful knowledge on modular solution elements that enables them to directly translate individual structural task features into characteristics of the problem solution. This knowledge might be much more helpful than conventional knowledge on problem categories and solution recipes for adapting solution procedures to novel problems beyond the known problem categories (cf. Catrambone, 1998).

When we considered how to 'rethink' the domain of calculating complex event probabilities in order to develop instructional examples that do not refer to problem categories, their defining structural features, and category-specific solution formulas, we relied on the fact that problems in probability theory can be solved by breaking down complex events into sets of individual events. Accordingly, the calculation of a complex event probability by means of a formula can be decomposed into a sequence of simpler calculations, that is, calculations of individual event probabilities. In line with our approach, the calculation of individual event probabilities allows one to directly relate individual structural task features and individual characteristics of solution steps. This is not true for the molar approach of using a solution formula! The solution procedure based on the modular approach we developed comprises four steps that are illustrated in the worked example in the right column of Table 1 (the left column displays the conventional molar solution approach for solving the same task). In this example the probability of a complex event is 
calculated by determining the probabilities of all individual events that make up the complex event (steps 1 to 3 ) and then multiplying these individual event probabilities to calculate the overall probability (step 4).

When calculating a particular individual event probability one has to take into account how the number of possible and acceptable choices changes from the preceding to the current trial. These changes depend on whether previously selected objects are replaced or not after having been selected (problem with or without replacement), and on whether there is more than one acceptable choice in a given trial (order of selection important or not). For problems without replacement the number of possible choices decreases from trial to trial (otherwise it remains the same). For problems in which the order of selection is important there is only one acceptable choice on each trial (otherwise there might be more than one acceptable choices).

The fact that the two structural features used to categorize problems in this domain correspond to particular characteristics of individual solution steps makes it easier to adapt the modular approach to novel problems. The solution procedure illustrated by the modular example format does not require one to categorize problems before solving them. Rather, decisions with regard to individual structural task features can be directly translated into modifications of individual solution steps (i.e., changes in the number of possible and acceptable choices from trial to trial). The reasoning exemplified in the modular example format thus should help learners to understand relations below the category level that hold irrespective of category membership. What is even more important - in contrast to the category-based approach -- is that this reasoning can be understood by holding only a rather limited amount of information in working memory simultaneously. Thus, this format should impose less intrinsic cognitive load than a molar example format and accordingly free cognitive resources that can then be used by learners to 
engage in profitable processes of elaborating and comparing example problems.

To sum up, compared to traditional molar examples, the alternative example format we constructed is modular because solution procedures are broken down into smaller meaningful solution elements that can be understood in isolation without holding large amounts of information active in working memory. These elements can be separately transferred when solving novel problems. In this respect, modular examples conform to the subgoal learning model that proposes to group sets of solutions steps according to the subgoals they aim to achieve in the solution procedure (Catrambone, 1998).

In the second part of this paper we will review the available evidence that an example format characterized by a modular presentation of solution procedures can reduce cognitive load, improve learning from worked examples, and foster transfer to novel problems.

\section{Review of Experimental Evidence}

In order to evaluate the evidence for the claimed superiority of a modular example format we can refer to five studies that are reported in Catrambone (1994), Gerjets, Scheiter, and Kleinbeck (in press), and Gerjets, Scheiter, and Catrambone (in press). An overview of these studies will be given in the following sections.

Study 1 (Catrambone, 1994)

In Experiment 1 of Catrambone (1994), 66 learners studied a pair of worked examples in which both examples belonged to the same single problem category (permutation without replacement). Each worked example demonstrated how to calculate a complex event probability that was related to humans picking a particular configuration of objects by chance. Learners were asked to study carefully the booklet with the two examples at their own pace and they were told that they would be asked later to solve some problems without looking at the examples. After 
studying, learners wrote a description of how to solve problems in the domain. Finally, they solved four test problems that either belonged to the same problem category as the learning examples (isomorphic problems: permutations without replacement) or to a different problem category (novel problems: combinations without replacement). The experimental manipulation was whether the two worked examples for learning were designed to be molar or modular. The molar examples explained the appropriate solution formula for solving the problems and demonstrated how to insert the correct variable values into the formula. The modular examples explained how to consider the complex event in question as a sequence of individual events and how to integrate individual event probabilities into an overall complex event probability. The modular-example group outperformed the molar-example group on transfer performance. These results provided initial evidence that modular examples might help to foster learning from worked examples.

Study 2 (Catrambone, 1994)

In Experiment 2 of Catrambone (1994) the findings of Study 1 where replicated under slightly different conditions. To further improve learning the participants $(\mathrm{N}=78)$ received three worked examples of a single problem category (permutation without replacement) in the learning phase. Participants were not required to describe the solution procedure before solving the test problems as they were in Study 1. Finally, the wording of the test problems was slightly modified. The results of Study 2 were similar to those from Study 1 in that learners who received modular examples clearly outperformed the molar group in transfer performance.

However, both of the initial studies of Catrambone (1994) demonstrating the superiority of a modular example format were characterized by two limitations with regard to the claims elaborated in the current paper. First, no measurement of learning time and no measurement of 
cognitive load was administered; thus we do not know whether the molar and modular example formats differ in their processing demands as can be expected from the theoretical considerations outlined in this paper. Second, the experiments did not address the ecologically more natural situation of learners studying multiple problem categories in a domain. The latter concern may easily result in an artificial bias in favor of the modular example format: When students are confronted with multiple problem categories during learning and problem solving they might strongly profit from being able to distinguish among these categories with regard to their structural task features, an ability that might be better supported by molar examples. Thus, the initial results of Catrambone (1994) might be valid only in the rather restricted situation in which learning only one problem category is required.

Study 3 (Gerjets, Scheiter, \& Kleinbeck, in press)

The first experiment reported in Gerjets, Scheiter, and Kleinbeck (in press) changed the design of Study 1 and 2 used in Catrambone (1994) in that learners had to acquire multiple problem categories by using a nonlinear hypertext learning environment that allowed the experimenters to measure the time for learning and later problem solving by means of logfiles. Time parameters were used to measure the processing demands of the two different example formats. In this experiment 52 learners could study two example problems for each of six different problem categories related to calculating complex event probabilities. These examples contained instructional explanations as illustrated in Table 1. The examples could be retrieved by means of hyperlinks in the learning environment used for self-paced study. In the introductory instructions of the experiments, participants were informed that they would have to solve six probability test problems on their own after having studied the worked examples. To avoid memory artifacts - in particular in the molar group that had to remember six different solution 
formulas - a re-examination of the instructional examples during the test phase was possible. In the test phase every participant had to solve three isomorphic and three novel test problems. Isomorphic test problems differed from the instructional examples only with regard to their surface features, whereas novel test problems were constructed in a way that two complex event probabilities had to be found and then multiplied in order to calculate the required probability.

Logfiles were used to measure example-study time, time for retrieving instructional examples during problem solving (re-examination time), and time for solving the test problems. As a performance measure the percentage of correctly solved test problems was registered. A declarative pretest was used to distinguish between high and low prior-knowledge learners within the two groups learning with molar or modular examples. Prior knowledge was used as an additional independent variable because it is strongly related to intrinsic cognitive load according to the cognitive load theory (cf. Sweller et al., 1998).

The results of Study 3 showed that learning with modular examples led to better problemsolving performance for isomorphic as well as novel problems irrespective of learners' level of prior knowledge (cf. Figure 1). Additionally, learning with modular examples required significantly less example-study time as well as re-examination time during problem solving. No differences with regard to problem-solving time were obtained.

\section{--- Insert Figure 1 here -----}

In sum, Study 3 demonstrated that a modular example format is not only superior when a single problem category has to be acquired but also in case of multiple problem categories. This finding rules out the concern from Study 1 and 2 (Catrambone, 1994) that the superiority of a modular example format might be an artifact due to the restricted learning situation used. It seems that 
students' ability to distinguish among different problem categories with regard to their structural task features will not be better supported by molar examples than by modular examples although molar examples are explicitly designed to convey knowledge on problem categories and their structural task features. To explore this issue in greater detail we conducted a replication of Study 3 that used a slightly different instruction and that contained two additional dependent measures.

Study 4 (Gerjets, Scheiter, \& Kleinbeck, in press)

The second experiment reported in Gerjets, Scheiter, \& Kleinbeck (in press) used exactly the same procedure as in Study 3 (including the same set of worked examples and test problems). However, a different task was announced to learners at the beginning of the experiment. Instead of telling participants that they would have to solve test problems later on, they were instructed to study the instructional examples with the goal of acquiring structural features of problem categories. Participants were informed that they would have to work on a classification task and on a comparison task after having studied the instructional materials. They were made aware of the importance of knowing about structural features of different problem categories by familiarizing them with these two tasks they later would have to accomplish in the test phase.

Classification task: To accomplish this task, participants had to identify one out of six word problems that was most similar to a given test problem with regard to its structural features. Four of the instructional examples that were already known from the learning phase were used as test problems. For each of the four test problems six word problems were presented as multiple-choice items that differed with regard to the problem category they belonged to. For each test problem participants had to identify the structurally most similar word problem, that is, the word problem that belonged to the same problem category as the test problem. 
Comparison task: In this task, participants had to compare pairs of word problems with regard to their structural similarities and differences. Six problems from different problem categories were used as test problems. These problems were already known from the learning phase. From the 15 possible problem pairs that could have been made up from these six problems, we selected six problem pairs that were presented to participants for the comparison task. For each pair participants had to fill in a form that asked for structural similarities and differences of the two word problems.

Following the learning phase participants had to accomplish three different tasks. First they had to solve the same test problems as in Study 3 without having been informed about this task in advance. Subsequently, they had to work on the two tasks that had been announced and explained to them at the beginning of the experiment. The same time data as in Study 3 were obtained by means of logfiles.

The results of Study 4 were similar to those of Study 3 and again supported the claim that learning with modular examples led to better problem-solving performance for isomorphic as well as novel problems irrespective of learners' level of prior knowledge (cf. Figure 2). Again, learning with modular examples required significantly less example-study time as well as less reexamination time during problem solving. No differences with regard to problem-solving time were obtained.

\section{--- Insert Figure 2 -----}

With regard to the comparison task and the classification task that were used to investigate the influence of example formats and prior knowledge on the acquisition of structural features of problem categories, we found that performance in the classification task was affected only by participants' prior knowledge. Both example formats were equally effective in conveying 
knowledge on structural features of problem categories necessary to solve the classification task. The same pattern of results were obtained for the comparison task.

These findings can be seen as indicating that learners might experience substantial difficulties in extracting and understanding information on abstract structural task features and problem categories from molar worded examples although these examples are explicitly designed to convey that information. In line with our initial cognitive load analysis, learners studying molar worked examples might have few cognitive resources left to engage in profitable processes of example elaboration and example comparison which are, however, often shown to be necessary to construct more abstract knowledge from specific example problems.

An explanation might be that the understanding of molar example solutions requires learners to hold a substantial amount of information simultaneously in working memory. In contrast, understanding modular example solutions might be less demanding for students so that they are able to develop an understanding of structural problem features and to infer the categorical structure of the domain by themselves without being explicitly provided with this information. Accordingly, they accomplished the same level of performance in the classification task and the comparison task that were used to measure this particular aspect of students' knowledge. In our view, this can be seen as evidence that students learning from modular worked examples may possess unused cognitive resources that might be invested in (germane) processes that are directly relevant to learning and understanding (e.g., self-explanations).

Study 5 (Gerjets, Scheiter, \& Catrambone, in press)

To provide more direct evidence for the line of reasoning outlined above, we conducted another experiment (described as Experiment 2 in Gerjets, Scheiter, and Catrambone, in press) that involved a cognitive load measurement in order to test whether the cognitive load is indeed 
lower for studying modular example solutions than for studying molar example solutions. Beyond including measures of cognitive load we manipulated the availability of instructional explanations in Study 5. Two competing hypotheses can be formulated with regard to the impact of instructional explanations: On the on hand, learners using the modular example format might have sufficient cognitive resources available to engage in self-explanations, whereas learners using the molar example formats might suffer from cognitive overload when trying to understand molar solution procedures. Accordingly, students learning with modular examples might not need highly elaborated examples compared to students learning with molar examples. On the other hand, the substantial amount of instructional explanation provided in Study 3 and 4 (cf., Table 1) might have imposed cognitive load on learners that is especially harmful for those studying molar worked examples. According to this line of reasoning, learners with molar examples might benefit from a more condensed and therefore less overwhelming presentation of solution procedures, whereas learning from modular examples might be less vulnerable to cognitive overload due to the provision of instructional examples. This ambiguity with regard to the expected effectiveness of instructional explanations is consistent with the existing literature on this instructional manipulation (cf. Renkl, 2002).

In this experiment 68 students from the Georgia Institute of Technology learned with a shortened and linearized version of the learning environment used in Study 3 and 4 (in order to reduce navigational demands). Learners were provided with two worked examples for each of four problem categories taught. After studying the examples participants solved three isomorphic and six transfer problems. Novel problems were constructed similarly to the ones used in Study 3 and 4 (Gerjets, Scheiter, \& Kleinbeck, in press). In contrast to these experiments, however, participants were given no opportunity to re-examine instructional examples in the test phase. 
Instead, solution formulas were provided during problem solving for those participants who had to apply these formulas (i.e., those learning with molar examples). The formula list was provided during problem solving in order to allow direct access to the solution formulas and to rule out the possibility that a potential inferiority of the molar example format may be traced back to participants merely forgetting formula details (or having difficulties in finding the appropriate formula when re-examining instructional examples).

As in the previous studies, the worked examples were either presented in the molar or the modular example format. Additionally, we varied the degree of instructional explanations between subjects. Half of the participants learned from the highly elaborated examples used in Study 3 and 4 while the other half studied a rather condensed version of the examples. Whereas the highly elaborated examples provided detailed justifications for solution steps, the condensed examples focused on the mathematical structure of example solutions without providing instructional explanations (see Table 1).

As dependent variables, problem-solving performance for isomorphic and novel problems as well as example-study times and problem-solving times were recorded. In order to test the assumption that using modular examples would lead to a reduction of intrinsic cognitive load during learning, we additionally assessed different aspects of cognitive load after the learning phase by administering a modified version of the NASA-TLX questionnaire (Hart \& Staveland, 1988). We preferred the NASA-TLX to the usual cognitive load questionnaire introduced by Paas and van Merriënboer (1994) because it allows for a more detailed analysis. Each of the three cognitive load items was rated on a scale ranging from 0 (low cognitive load) to 100 (high cognitive load). The following subscales were used: 'Effort' (How hard did you have to work in your attempt to understand the contents of the learning environment?), 'Stress' (How insecure, 
discouraged, irritated, stressed, and annoyed did you feel during the learning task?), and 'Task demands' (How much mental and physical activity was required (e.g., thinking, deciding, calculating, remembering, looking, searching etc.)? Was the learning task easy or demanding, simple or complex, exacting or forgiving?).

Analyzing the cognitive load data revealed that the example formats influenced learners’ subjective experience of cognitive load whereas the degree of instructional explanations had no reliable impact (see Figure 3). First, with regard to the effort participants believed they had to invest in the task, they indicated that they had to work less hard in order to understand the instructional contents when learning with modular examples. Second, participants experienced far less stress during learning with modular examples. However, there was no effect of example format on the task demands associated with the learning task, but there was a significant interaction between example format and instructional explanations. The interaction indicated that participants judged the learning task as being less demanding with modular examples than with molar examples - but only when instructional explanations were provided - whereas there was no difference between the two examples formats when no explanations were given.

\section{--- Insert Figure 3 here ---}

As expected, participants who had learned with modular examples clearly outperformed participants learning with molar examples with regard to problem-solving performance for isomorphic as well as for novel problems (see Figure 4). There was, however, no effect of instructional explanations nor did any of the factors interact. Finally, analyzing the time data revealed that not only were participants learning with a modular example format more successful with regard to problem-solving performance, but they also needed far less time studying the 
examples than participants learning with molar examples. Rather naturally, the examples that included explanations took longer to process. There was no interaction between the two factors. As in the previous studies there were no effects with regard to problem-solving time.

--- Insert Figure 4 here ---

\section{Discussion}

Much of the existing research on learning from worked examples has been based on the notion of problem-type schemas as a central prerequisite for proficient problem solving. In this paper, we have argued that this conventional approach of designing examples in a way that focuses on teaching schemas might result in molar examples that impose high levels of cognitive load on learners. The cause for this problem might be that all the information that has to be integrated into the schema (e.g., multiple task features, formulas) has to be kept active in mind simultaneously. This high degree of cognitive load associated with molar, schema-based examples might prevent learners from implementing higher-level example processes like elaborations and comparisons. Instructional designers have addressed this problem by inventing a variety of techniques to either foster profitable processing of molar examples (and thereby increasing germane cognitive load) and/or to reduce unnecessary demands imposed on the learner (and thereby decreasing extraneous cognitive load).

In our work we took another approach by trying to design examples in a way that reduces the task-related, intrinsic load. Similar approaches of reducing intrinsic load have already yielded promising results recently (van Merriënboer et al., 2003). The basic idea of our modular approach was to break down solution procedures into smaller. more meaningful, pieces that can be conveyed and understood separately. In five studies we provided evidence that indeed these modular examples are superior to molar examples with regard to problem-solving performance 
for isomorphic and novel problems, different measures of learning time, and cognitive load.

The positive effects of modular examples were found to be superior regardless of the number of problem categories taught, the learning task announced, and the amount of instructional explanations given. Furthermore, modular examples proved to be superior for learners with low as well as with high prior knowledge. Therefore, the advantages of modular examples for teaching problem-solving skills seem to be very stable over a variety of instructional conditions.

One possible critique that we are aware of is that the design of modular versus molar examples might be restricted to this specific area of probability theory, that is, calculating complex event probabilities. We do not believe that this is the case; rather we are convinced that this approach might be extended to other well-structured domains where problems fall into categories. In fact, Catrambone (1994) has already successfully attempted to extend this approach to the domain of algebra word problems - a line of research that we would like to follow in our future work. Another possible critique might be that modular examples might be helpful only for calculating complex event probabilities when rather small numbers are involved, whereas the strength of using formulas comes into play for problems dealing with larger numbers. However, we are convinced that in order to be able to apply a formula to novel cases, a learner must have already achieved an understanding of the domain. Therefore, we recommend using modular examples for initial skill acquisition in order to foster this kind of understanding and to switch to a molar approach in later stages of skill development. Learners would have at that point sufficient prior knowledge available to cope with the complexity of the formulas, that is, for them even a molar approach might be characterized by a low level of element interactivity. That is, we do not propose that the solution procedures conveyed by means of modular and molar examples are mutually exclusive; rather it is the instructional designer's important and difficult task to decide 
when to quit a modular approach in favor of using formulas and to rely on learners' ability to categorize problems according to their structural features. This decision should be based on empirical evidence with regard to finding the most suitable transition point between these two approaches. However, this empirical evidence is not yet available and has to be obtained by future research.

\section{References}

Atkinson, R. K., \& Catrambone, R. (in press). Examining the use of conceptual equations and elaborations to aid transfer in statistical learning. Journal of Educational Psychology.

Atkinson, R. K., Derry, S. J., Renkl, A., \& Wortham, D. W. (2000). Learning from examples: Instructional principles from the worked examples research. Review of Educational Research, $70,181-214$

Catrambone, R. (1994). Improving examples to improve transfer to novel problems. Memory \& Cognition, 22, 606-615.

Catrambone, R. (1998). The subgoal learning model: Creating better examples to improve transfer to novel problems. Journal of Experimental Psychology: General, 127, 355-376.

Catrambone, R., \& Holyoak, K. J. (1989). Overcoming contextual limitations on problem-solving transfer. Journal of Experimental Psychology: Learning, Memory, and Cognition, 15, 11471156.

Chi, M. T. H., Bassok, M., Lewis, M., Reimann, P., \& Glaser, R. (1989). Self-explanations: How students study and use examples in learning to solve problems. Cognitive Science, 13, 145182.

Chi, M. T. H., de Leeuw, N., Chiu, M.-H., \& LaVancher, C. (1994). Eliciting self-explanations improves understanding. Cognitive Science, 18, 439-477. 
Cummins, D. D. (1992). Role of analogical reasoning in the induction of problem categories. Journal of Experimental Psychology: Learning, Memory, and Cognition, 18, 1103-1124.

Derry, S. J. (1989). Strategy and expertise in solving word problems. In C. B. McCormick, G. Miller, \& M. Pressley (Eds.), Cognitive strategy research: From basic research to educational applications (pp. 269-302). New York: Springer.

Gagné, R. M. (1962). The acquisition of knowledge. Psychological Review, 69, 355-365.

Gerjets, P., \& Scheiter, K. (2003). Goal configurations and processing strategies as moderators between instructional design and cognitive load: Evidence from hypertext-based instruction. Educational Psychologist, 38, 33-41.

Gerjets, P., Scheiter, K. \& Catrambone, R. (in press). Reducing cognitive load and fostering cognitive skill acquisition: Benefits of category-avoiding examples. In R. Alterman \& D. Kirsh (Eds.), Proceedings of the $25^{\text {th }}$ Annual Conference of the Cognitive Science Society. Mahwah, NJ: Erlbaum.

Gerjets, P., Scheiter, K., \& Kleinbeck, S. (in press). Instructional examples in hypertext-based learning and problem solving: Comparing transformational and derivational approaches to example design. In H. M. Niegemann, R. Brünken, \& D. Leutner (Eds.), Instructional design for multimedia learning. Muenster: Waxmann.

Gerjets, P., Scheiter, K., \& Tack, W. H. (2000). Resource-adaptive selection of strategies in learning from worked-out examples. In L. R. Gleitman \& A. K. Joshi (Eds.), Proceedings from the 22nd Annual Conference from the Cognitive Science Society (pp. 166-171). Mahwah, NJ: Erlbaum.

Gick, M. L., \& Holyoak, K. J. (1983). Schema induction and analogical transfer. Cognitive Psychology, 15, 1-38. 
Hart, S. G., \& Staveland, L. E. (1988). Development of NASA-TLX (Task Load Index): Results of experimental and theoretical research. In P. A. Hancock \& N. Meshkati (Eds.) Human Mental Workload (pp. 139-183). Amsterdam: North Holland.

Lawless, K. A., \& Brown, S. W. (1997). Multimedia learning environments: Issues of learner control and navigation. Instructional Science, 25, 117-131.

LeFevre, J., \& Dixon, P. (1986). Do written instructions need examples? Cognition and Instruction, 3, 1-30.

Paas, F. G. W. C. \& van Merriënboer, J. J. G. (1994). Variability of worked examples and transfer of geometrical problem solving skills: A cognitive load approach. Journal of Educational Psychology, 86, 122-133.

Pirolli, P., \& Recker, M. (1994). Learning strategies and transfer in the domain of programming. Cognition and Instruction, 12, 235-275.

Quilici, J. L., \& Mayer, R. E. (1996). Role of examples in how students learn to categorize statistics word problems. Journal of Educational Psychology, 88, 144-161.

Reed, S. K. (1993). A schema-based theory of transfer. In D. K. Detterman \& R. J. Sternberg (Eds.), Transfer on trial: Intelligence, cognition, and instruction (pp. 39-67). Norwood, NJ: Ablex.

Reed, S. K. (1999). Word problems. Mahwah, NJ: Erlbaum.

Reed, S. K., Dempster, A., \& Ettinger, M. (1985). Usefulness of analogous solutions for solving algebra word problems. Journal of Experimental Psychology: Learning, Memory, and Cognition, 11, 106-125.

Renkl, A. (1997). Learning from worked-out examples: A study on individual differences. Cognitive Science, 21, 1-29. 
Renkl, A. (1999). Learning mathematics from worked-out examples: Analyzing and fostering self-explanations. European Journal of Psychology of Education, 14, 477-488.

Renkl, A. (2002). Learning from worked-out examples: Instructional explanations supplement self-explanations. Learning \& Instruction, 12, 529-556.

Renkl, A. \& Atkinson, R. K. (2003). Structuring the transition from example study to problem solving in cognitive skills acquisition: A cognitive load perspective. Educational Psychologist, 38, 15-22.

Ross, B. H. (1989). Distinguishing types of superficial similarities: Different effects on the access and use of earlier problems. Journal of Experimental Psychology: Learning, Memory, and Cognition, 15, 456-468.

Schuh, J., Gerjets, P., \& Scheiter, K. (2003). Supporting learning from worked-out examples in computer-based learning environments. In F. Schmalhofer, R. Young, \& G. Katz (Eds.), Proceedings of the European Cognitive Science Conference 2003 (pp. 301-306). Mahwah, NJ: Erlbaum.

Sowder, L. (1985). Cognitive psychology and mathematical problem solving: A discussion of Mayer's paper. In E. A. Silver (Ed.), Teaching and learning mathematical problem solving: Multiple research perspectives (pp. 139-146). Hillsdale, NJ: Erlbaum.

Sweller, J. (1994). Cognitive load theory, learning difficulty, and instructional design. Learning and Instruction, 4, 295-312.

Sweller, J., van Merriënboer, J. J. G., \& Paas, F. W. C. (1998). Cognitive architecture and instructional design. Educational Psychology Review, 10, 251-296.

VanLehn, K. (1989). Problem solving and cognitive skill acquisition. In M. I. Posner (Ed.), Foundations of cognitive science (pp. 527-579). Cambridge, MA: MIT Press. 
VanLehn, K. (1996). Cognitive skill acquisition. Annual Review of Psychology, 47, 513-539.

Van Merriënboer, J. J. G., Kirschner, P. A., \& Kester, L. (2003). Taking the load of a learner’s mind: Instructional design for complex learning. Educational Psychologist, 38, 5 - 13.

Van Merriënboer, J. J. G. (1992). Strategies for programming instruction in high school: Program completion vs. program generation. Journal of Educational Computing Research, 6, 265-287. 
Table 1

Molar and Modular Example Formats Used for Experimentation

\section{M-SPRINT EXAMPLE}

At the Olympics 7 sprinters participate in the $100 \mathrm{~m}$-sprint. What is the probability of correctly guessing the winner of the gold, the silver, and the bronze medals?

MOLAR EXAMPLE FORMAT
IDENTIFY TASK FEATURES
This problem is a permutation-without-replacement
problem. Problems of this type have two important
features: First, the order of selection is important, and
second, there is no replacement of selected elements.
We are not interested only in finding out just which 3 of
the 7 sprinters win medals, rather we want to know
specifically which sprinter wins which medal. Therefore,
the order of selection matters. A sprinter can win at most
only one medal. Thus, this problem is a problem without
replacement. That is, after a sprinter wins a medal he is
not eligible for being selected again.

\section{APPLY FORMULA}

For this type of problem the following formula should be applied: $\mathbf{A}=\mathbf{n} ! /(\mathbf{n}-\mathbf{k})$ ! with $n$ being the number of all sprinters and $k$ being the number of sprinters that have to be correctly guessed.

\section{INSERT VALUES}

In the given example there are 7 sprinters to choose from. This is the set of elements for selection $(n=7)$. As we want to find out the probability of correctly guessing the winner of the gold, the silver, and the bronze medals, 3 sprinters out of these 7 sprinters have to be selected. Therefore, the number of selected sprinters equals $\mathrm{k}=3$. Inserting these values into the formula for permutation without replacement yields 7! / (7- 3)! = 210 possible permutations.

\section{CALCULATE PROBABILITY}

In order to calculate the probability of correctly guessing the winner of each of the three medals, divide 1 (the particular permutation we are interested in) by the number of possible permutations. Thus, the probability of getting this permutation (the winner of each of the three medals) equals $\mathbf{1} / \mathbf{2 1 0}$.

\section{MODULAR EXAMPLE FORMAT}

FIND 1ST EVENT PROBABILITY

In order to find the first event probability you have to consider the number of acceptable choices and the pool of possible choices. The number of acceptable choices is 1 because only 1 sprinter can win the gold medal. The pool of possible choices is 7 because 7 sprinters participate in the $100 \mathrm{~m}$-sprint. Thus, the probability of correctly guessing the winner of the gold medal is $\mathbf{1 / 7}$.

\section{FIND 2ND EVENT PROBABILITY}

In order to find the second event probability you again have to consider the number of acceptable choices. The number of acceptable choices is still 1 because only 1 sprinter can win the silver medal. The pool of possible choices is reduced to 6 because only the remaining 6 sprinters participating in the $100 \mathrm{~m}$-sprint are eligible to receive the silver medal. Thus, the probability of correctly guessing the winner of the silver medal is $\mathbf{1 / 6}$.

\section{FIND 3RD EVENT PROBABILITY}

In order to find the third event probability you again have to consider the number of acceptable choices. The number of acceptable choices is still 1 because only 1 sprinter can win the bronze medal. The pool of possible choices is reduced to 5 because only the remaining 5 sprinters participating in the $100 \mathrm{~m}$-sprint are eligible to receive the bronze medal. Thus, the probability of correctly guessing the winner of the bronze medal is $\mathbf{1 / 5}$.

\section{CALCULATE THE OVERALL PROBABILITY}

The overall probability is calculated by multiplying all individual event probabilities. Thus, the overall probability of correctly guessing the winner of each of the three medals is $1 / 7 * 1 / 6 * 1 / 5=1 / 210$.

Note: In experimental conditions with instructional explanations the example solutions contained all information stated in the relevant table column. Conditions without instructional explanations contained only the information printed in bold. 
Figure Caption(s)

Figure 1: Problem-Solving Performance (\% Correct) for Isomorphic and Novel Problems, Example-Study Time, Re-examination Time, and Problem-Solving Time (in Seconds) for Isomorphic and Novel Problems as a Function of Example Format and Prior Knowledge

Figure 2: Problem-Solving Performance (\% Correct) for Isomorphic and Novel Problems, Example-Study Time, Re-examination Time, and Problem-Solving Time (in Seconds) for Isomorphic and Novel Problems as a Function of Example Format and Prior Knowledge

Figure 3: Cognitive Load (Scale Values) as a Function of Example Format and Degree of Instructional Elaborations

Figure 4: Problem-Solving Performance (\% Correct) for Isomorphic and Novel Problems, Example-Study Time, and Problem-Solving Time (in Seconds) as a Function of Example Format and Degree of Instructional Elaborations 

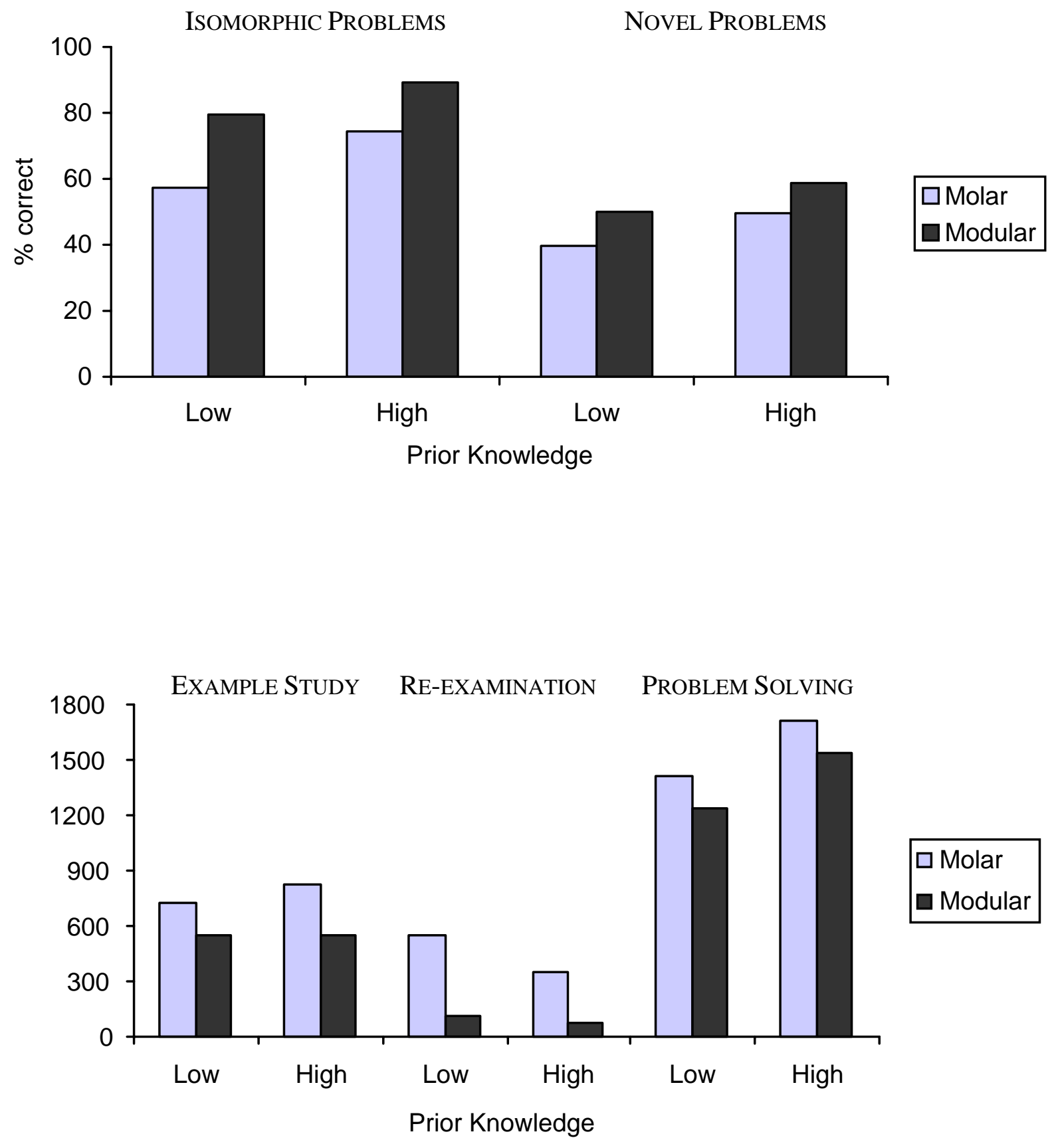

Figure 1 

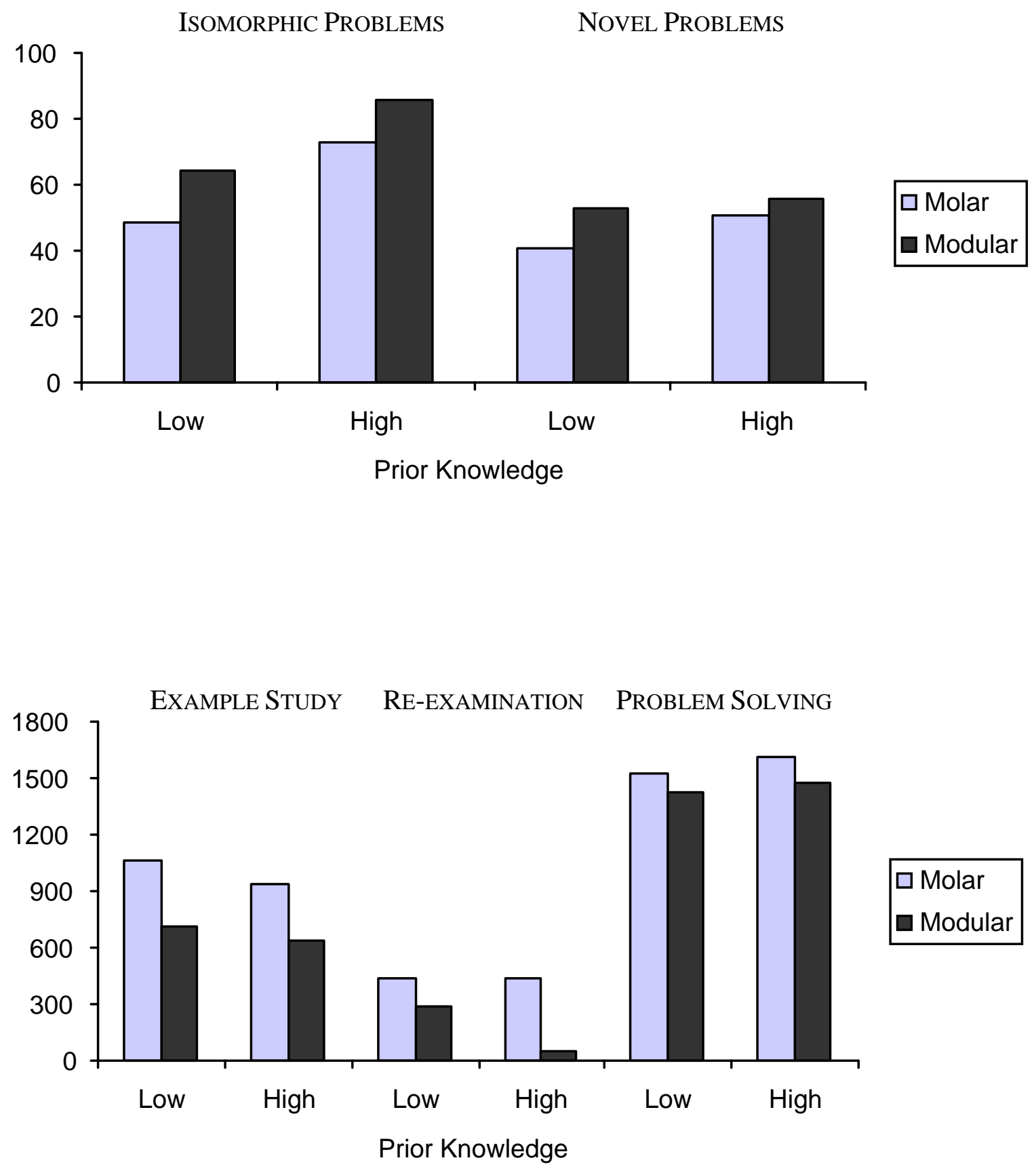

Figure 2 


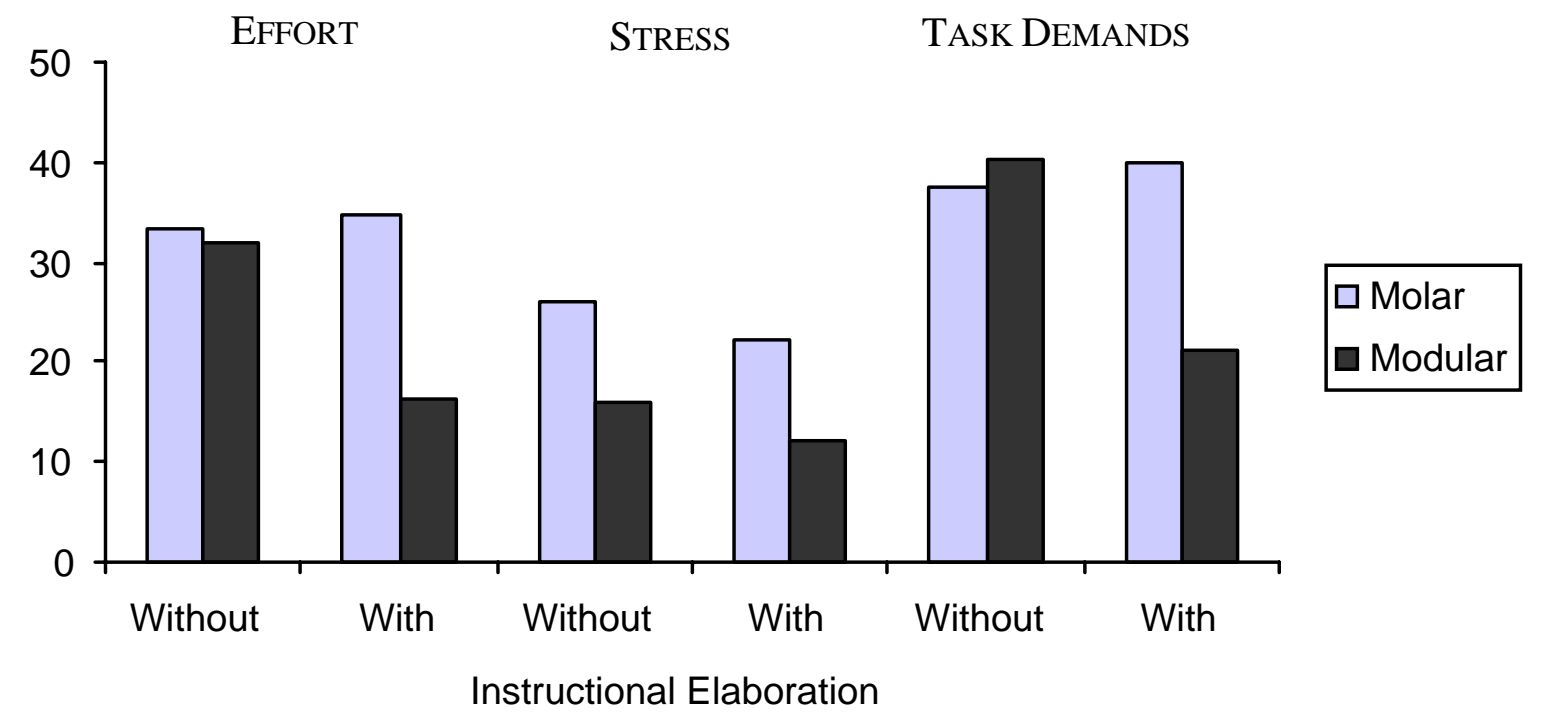

Figure 3 

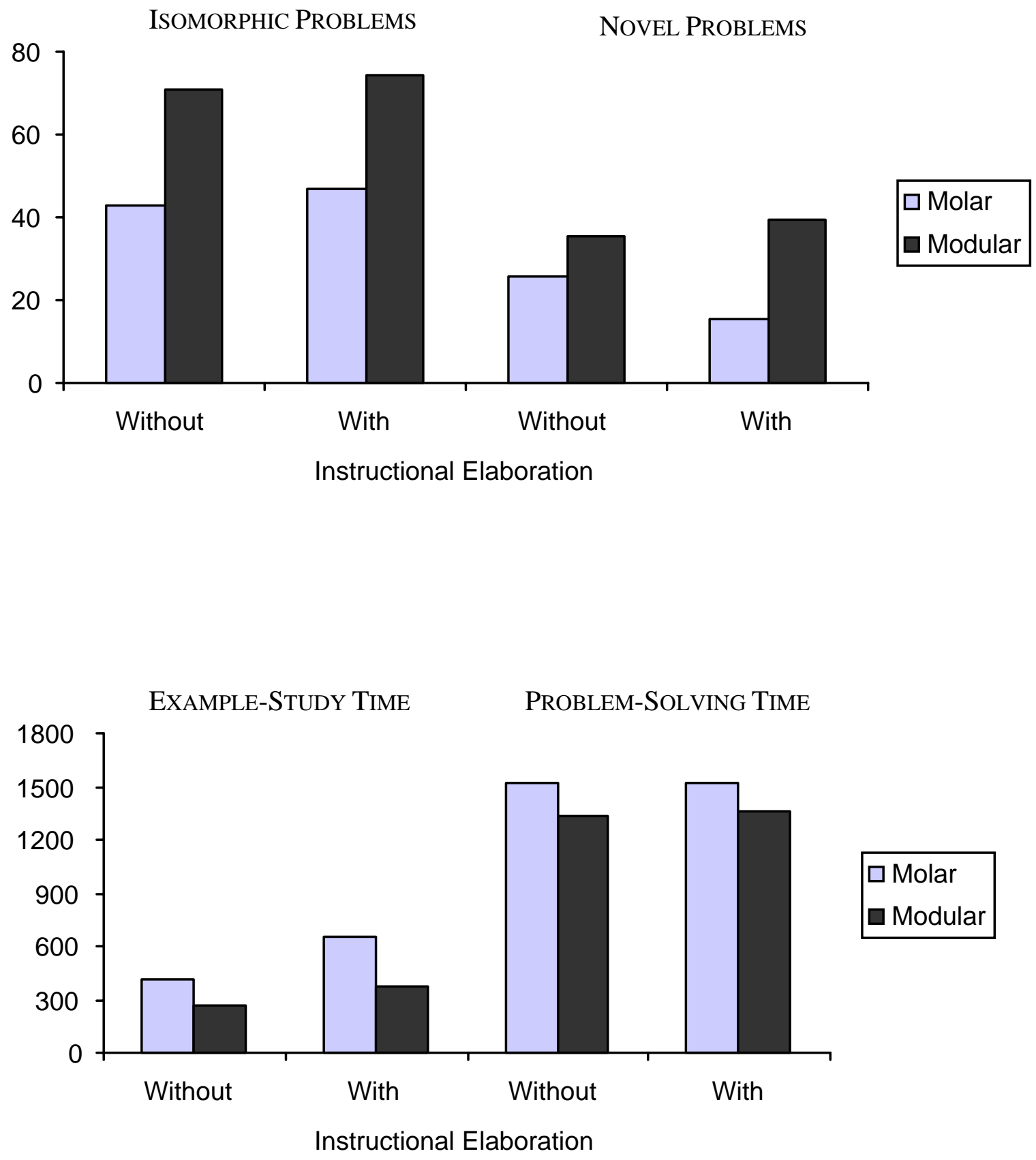

Figure 4 